\title{
BIG DATA, BUSINESS ANALYTICS AND BANK DIRECT MARKETING
}

Manying Qiu, Virginia State University, Petersburg, Virginia, U.S.A. Shuting Xu, Georgia Gwinnett College, Lawrenceville, Georgia, U.S.A. Yaquan Xu, Georgia Gwinnett College, Lawrenceville, Georgia, U.S.A.

\author{
dx.doi.org/10.18374/IJBR-17-3.3
}

\begin{abstract}
The global academic community has been continuously interested in using data mining techniques to improve the effectiveness of bank direct marketing campaign. Nowadays, consumers are empowered by digital devices. This paper analyzes the Portuguese bank's direct marketing campaign 2008-2013 data as an example to show that financial and economic information in the big data may influence clients' responses to the campaign. Automatic machine learning tools can assist practitioners to gain business insights effectively and efficiently.
\end{abstract}

Keywords: Data mining, predictive analytics, big data, business insights, direct marketing 